\title{
ANN Based Multi-classification Using Various Signal Processing Techniques for Bearing Fault Diagnosis
}

\author{
Chenxi Wu, Tefang Chen, Rong Jiang, Liwei Ning, Zheng Jiang \\ ${ }^{1}$ School of Information Science and Engineering, Central South University, \\ Changsha 410075, PR China \\ ${ }^{2}$ School of Mechanical Engineering, Hunan Institute of Engineering, Xiangtan \\ 411101, PR China \\ ${ }^{3}$ School of Information Science and Engineering, Wuhan University of Science \\ and Technology, Wuhan 430081, PR China \\ tefangchen@163.com
}

\begin{abstract}
Time domain features are employed for detection and identification of rolling element bearing faults in rotating machinery. Only five features with simple calculation are selected as features extracted directly from the original time domain vibration signals or preprocessed time domain vibration components. Three preprocessing techniques including high and band pass filtration, wavelet package transform (WPT) and envelope analysis are researched to achieve time domain features carrying the important diagnostic information of bearing conditions. An optimized artificial neural network (ANN) with rapid learning algorithm is designed and classification is performed using the ANN combined with time domain features. The model was evaluated on vibration data recorded using two accelerometers mounted on an induction motor housing subjected to a number of single point defects. The results demonstrate the proposed model is capable of high precision, fast processing and time savings in identification of bearing faults.
\end{abstract}

Keywords: Time Domain Features; ANN; Classification; Bearing Faults

\section{Introduction}

Rotating machine has been regarded as one of the essential systems on account of its extensive industrial applications. In order to find incipient fault, maintain a low downtime, and guarantee machine reliability and health trend, it is significant to detect different sorts of faults that probably occur in a machinery system like rotor rub, mass unbalance, shaft misalignment, gear failures and bearing defects. Generally speaking, techniques developed for fault detection and diagnosis mainly concentrated on three aspects: direct measurement of vibration by proper sensors, sound pressure level measurement using acoustic transducers and motor current signature analysis [1-6]. In the present work, vibration signatures were selected to detect bearing conditions in view of its convenient measurement and plentiful contents.

Although condition identification is possible by comparing the vibration signals of a machine with and without faulty bearings, the mixture of a variety of noise and the bearing resonant low-amplitude high-frequency broadband signals modulated by the impulses arising out of the low-frequency bearing defects poses trouble in detection of bearing condition using direct vibration signals. Thus, it is required to extract features from original or preprocessed signals related to bearing conditions. Features are then used to assess the performance of bearing. 
Theoretically, different faults have different frequency spectrum distributions, so faults can be recognized by analyzing the characteristic defect frequency (CDF). The CDFs of vibration signals in broken motors were probed to classify faults based on the relationship between amplitude of feature frequencies and fault types [7]. Feature frequencies of the rolling bearings were utilized as inputs of neural network to identify bearing defect types in motorbearing system [8]. Fast Fourier transform (FFT) of intrinsic mode functions (IMFs) decomposed using Hilbert-Huang transform (HHT) method were utilized to improve efficiency just using Hilbert transform (HT) in frequency domain for bearing fault diagnosis [9]. However, the relation matrix of feature frequencies and fault types is far more complex. There are some of major concerns that would bring about scope or error in diagnosis of bearing faults. First, because the impact vibration produced by bearing failure has relatively low energy, it is often masked by other interfering signals. Next, the existence of frequency sideband distributions originating from the variation of bearing dimensions, shaft speed and defect location makes it difficult to recognize the true CDF. Furthermore, the diagnosis results rely on expert's knowledge, experience and rules pre-defined, which probably gets inconsistent results to the same condition because each expert has his own judgment $[10,11]$.

On the other hand, some techniques based time domain analysis have been exploited to lowered or rule out these mentioned limitations on frequency domain analysis [12]. In [13], owing to its filtration and noise suppression ability in signal processing, the autoregressive (AR) model was constructed between time difference and vibration amplitude, the differences of AR coefficients then as feature inputs to the ANN for diagnosis of mass unbalance, misalignment, bearing defect and oil whip in rotating machinery. Standard deviation of wavelet packet coefficients as the feature vector of a more efficient ANN-based system was proposed to determine the type of gear failure [14]. The obtained results by using time domain features with the ANN turned out robust against speed or load variations in detection of rolling element bearing [15]. Time domain features were adopted to detect induction motors bearings conditions through the ANN, which were responsible for both lower computation and more precise fault diagnosis [16].

In the present work, a model is presented to make the diagnostics more efficient and reliable using time domain features as inputs to the ANN for bearing condition identification. The time domain features are achieved from the parts of the experimental dataset for known machine conditions. Some preprocessing techniques like high or band-pass filtering, wavelet packet transform and envelope analysis are investigated for feature extraction. The ANN is composed of five input nodes, one hidden layer with five nodes and one output layer with four nodes. Each of five input nodes represents root mean square, variance, skewness, kurtosis and normalized sixth central moment of the analyzed vibration signals, respectively. Four output nodes specify different bearing conditions: healthy, outer race defect, inner race defect and ball defect. The ANN is trained with back propagation algorithm and tested using the time domain features. The test results reveal the effectiveness of the proposed method in classification of bearing conditions.

The remaining sections of the paper are organized as follows: Section 2 describes the vibration signature in time domains with statistical parameters. Section 3 illustrates the high speed convergence algorithm for training the ANN needed less time and epochs. Section 4 formulates specific architectures of the ANN as a classifier. Section 5 includes various processing techniques and assessments about diagnostic results of bearing faults followed by the conclusions in Section 6. 


\section{Feature Selection}

The dataset for the proposed approach were taken from the Case Western Reserve University Bearing Data Center Website. The test bench consists of a 2 horsepower (HP) motor, a torque transducer, a dynamometer, and control electronics. The test bearings support the motor shaft. Four kinds of bearing conditions, namely, drive end bearings with healthy, outer race defect, inner race defect and ball defect were considered. Single point defect with diameter of 0.021 inches was introduced at the outer raceway, inner raceway and ball using electro-discharge machining, respectively. Two accelerometers were installed at both drive end and fan end of the motor housing. Thus, two channel signals were collected under the same bearing condition. The sampling frequency was $48,000 \mathrm{HZ}$ at speed of $1730 \mathrm{RPM}$ under 3 HP loading.

Two channel signals each with 20480 points were analyzed for each bearing condition. A channel signal had been separated into 20 segments of 1024 points. Five time domain features were extracted from each segment, which was defined as below:

(1) Root mean square

$$
R M S=\sqrt{\frac{\sum_{j=1}^{n}\left(x_{j}^{i}-u^{i}\right)}{n}}
$$

Where $n=1024$, is the number of each segment, $x_{j}^{i}$ is the jth sampling point of the ith segment, $i=1,2, \ldots, 20, j=1,2, \ldots, n$, and $u^{i}$ is the mean value of the ith segment.

$$
\mu^{i}=\frac{1}{N} \sum_{j=1}^{N} y_{j}^{i}
$$

(2) Variance

$$
\sigma^{2}=\frac{1}{n-1} \sum_{j=1}^{n}\left(x_{j}^{i}-u^{i}\right)^{2}
$$

(3) Skewness

$$
\gamma_{3}=\frac{1}{(n-1) \sigma^{3}} \sum_{j=1}^{n}\left(x_{j}^{i}-u^{i}\right)^{3}
$$

(4) Kurtosis

$$
\gamma_{4}=\frac{1}{(n-1) \sigma^{4}} \sum_{j=1}^{n}\left(x_{j}^{i}-u^{i}\right)^{4}
$$

(5) Normalized sixth central moment

$$
\gamma_{6}=\frac{1}{(n-1) \sigma^{6}} \sum_{j=1}^{n}\left(x_{j}^{i}-u^{i}\right)^{6}
$$

The five features were normalized to solve the signals variation in magnitude due to load or speed fluctuation and shorten training time of the ANN. For normalization, each feature was divided by the corresponding maximum value. These normalized features were in the range of 0 to 1 except the skewness between -1 and 1 . Figure 1 shows the plots of the 
normalized features of two channel signals, where each row represents the features of one channel signals containing four kinds of bearing conditions, and each column represents RMS, $\sigma^{2}, \gamma_{3}, \gamma_{4}$, and $\gamma_{6}$, respectively. There exists apparent difference among features; hence these features are proper for bearing fault diagnosis.
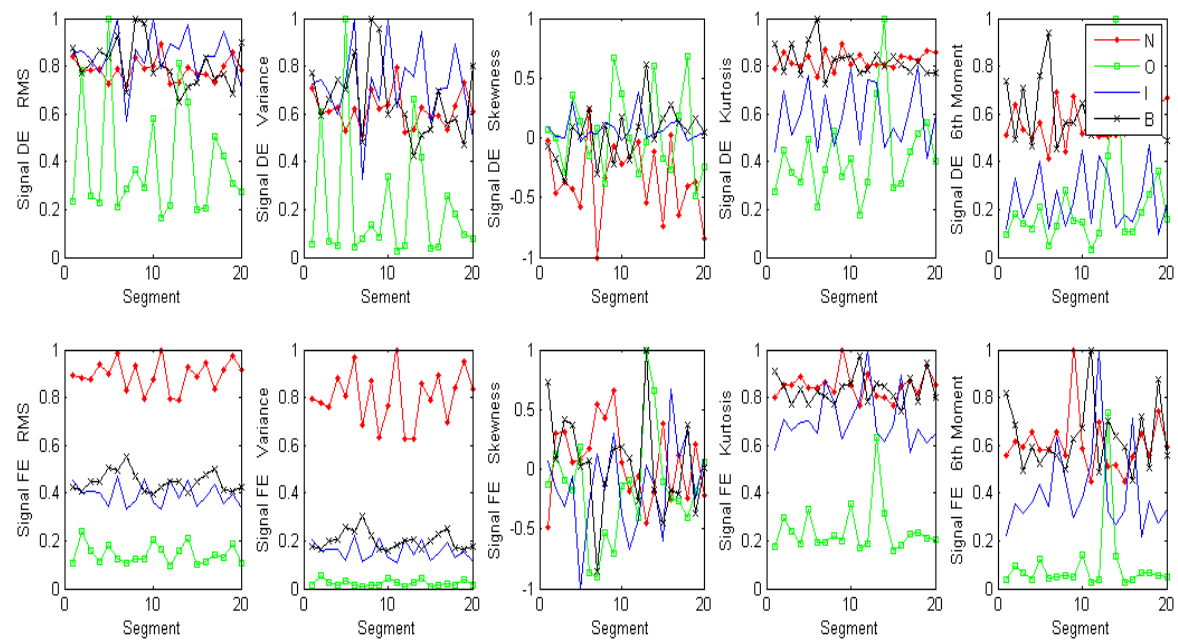

Figure 1. Time Domain Features of Acquired Vibration Signals: N, Normal; O: Outer Defect, I: Inner Defect, B: Ball Defect

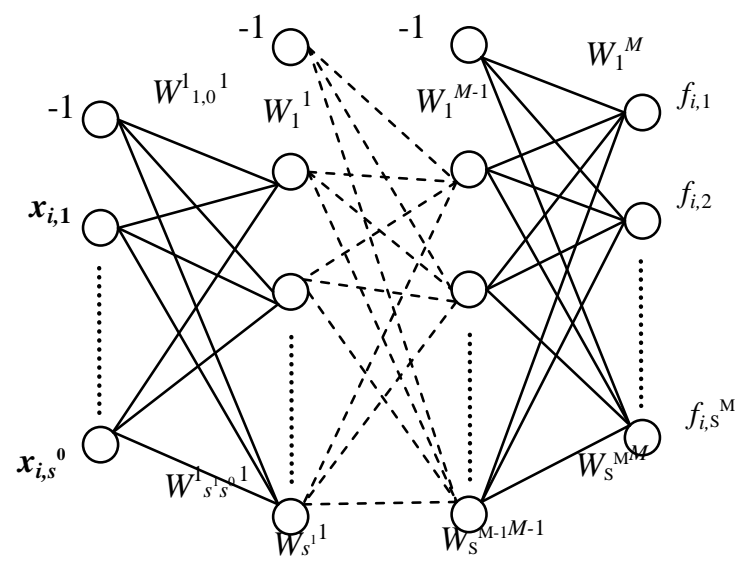

Figure2. Architecture of a M-layer ANN

\section{Training Algorithm}

Network training is proceeded by adjusting weight values based on a comparison of the output and the target until the network output matches the target. A multiple-layer network is shown in Figure 2, given the network performance index is the sum of the squares of the errors between the network output and the target

$$
F(\mathrm{w}(k))=\frac{1}{2} \sum_{i=1}^{Q} \sum_{j=1}^{s^{\mu}}\left(y_{i, j}-f_{i, j}\left(x_{i}, \mathrm{w}(k)\right)\right)^{2}
$$


Where $\mathrm{Q}$ is the total number of training pattern, $\mathrm{s}^{\mathrm{M}}$ is the number of output, $\mathrm{y}_{\mathrm{i}, \mathrm{j}}$ is the jth target output corresponding to the ith input vector, $\mathrm{f}_{\mathrm{i}, \mathrm{j}}\left(\mathrm{x}_{\mathrm{i}}, \mathrm{w}(\mathrm{k})\right)$ is the jth network output after the ith input vector, $\mathrm{w}(\mathrm{k})$ is a vector of network weights of the kth iteration and defined as

$$
\begin{aligned}
& \mathrm{w}(k)=\left[\begin{array}{lllllllllllllll}
\mathrm{w}_{1}^{1}(k)^{T} & \mathrm{w}_{2}^{1}(k)^{T} & \ldots & \mathrm{w}_{{ }^{\prime}}^{1}(k)^{T} & \mathrm{w}_{1}^{2}(k)^{T} & \mathrm{w}_{2}^{2}(k)^{T} & \ldots & \mathrm{w}_{s^{2}}{ }^{2}(k)^{T} & \ldots & \mathrm{w}_{1}{ }_{1}^{M}(k)^{T} & \mathrm{w}_{2}{ }_{2}(k)^{T} & \ldots & \mathrm{w}_{s^{\prime \prime}}{ }^{\prime \prime}(k)^{T}
\end{array}\right] \\
& \mathbf{w}_{1}^{m}(k)=\left[\begin{array}{lllll}
w_{1,0}^{m}(k) & w_{1,1}^{m}(k) & w_{1,2}^{m}(k) & \ldots & w_{1, s^{m-1}}^{m}(k)
\end{array}\right]^{T} \\
& \mathrm{w}_{2}^{m}(k)=\left[\begin{array}{lllll}
w_{2,0}^{m}(k) & w_{2,1}^{m}(k) & w_{2,2}^{m}(k) & \ldots & w_{2, s^{m-1}}^{m}(k)
\end{array}\right]^{T}
\end{aligned}
$$

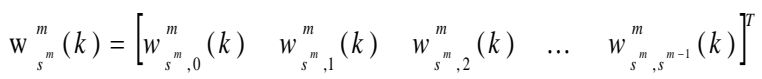

Note that the row indices on the elements of matrix $\mathrm{W}$ indicate the destination neuron of the weight, the column indices indicate which source is the input for that weight, and $\mathrm{m}$ is the number of network layer, $\mathrm{m}=1,2, \ldots, \mathrm{M}$.

The Taylor expansion of $\mathrm{f}_{\mathrm{i}, \mathrm{j}}\left(\mathrm{x}_{\mathrm{i}}, \mathrm{w}(\mathrm{k})\right)$ is

$$
f_{i, j}\left(x_{i}, \mathrm{w}(k)+\Delta \mathrm{w}(k)\right)=f_{i, j}\left(x_{i}, \mathrm{w}(k)\right)+J_{i, j} \Delta \mathrm{w}(k)+O\left(\|\Delta \mathrm{w}(k)\|^{2}\right) \approx f_{i, j}\left(x_{i}, \mathrm{w}(k)\right)+J_{i, j} \Delta \mathrm{w}(k)
$$

Where $J_{i, j}(\mathrm{w}(k))=\left[\begin{array}{llll}\frac{\partial f_{i, j}\left(x_{i}, \mathrm{w}(k)\right)}{\partial \mathrm{w}^{1}(k)} & \frac{\partial f_{i, j}\left(x_{i}, \mathrm{w}(k)\right)}{\partial \mathrm{w}^{2}(k)} & \ldots & \frac{\partial f_{i, j}\left(x_{i}, \mathrm{w}(k)\right)}{\partial \mathrm{w}^{n}(k)}\end{array}\right] \quad$ is the first partial derivatives (rowvector) of $f_{i, j}\left(x_{i}, w(k)\right)$ with respect to $w(k)$.

The approximation of $\mathrm{f}_{\mathrm{i}, \mathrm{j}}\left(\mathrm{x}_{\mathrm{i}}, \mathrm{w}(\mathrm{k})+\triangle \mathrm{w}(\mathrm{k})\right)$ gives

$$
F(\mathrm{w}(k)+\Delta \mathrm{w}(k))=\frac{1}{2} \sum_{i=1}^{Q} \sum_{j=1}^{s^{n}}\left(y_{i, j}-f_{i, j}\left(x_{i}, \mathrm{w}(k)+\Delta \mathrm{w}(k)\right)\right)^{2} \approx \frac{1}{2} \sum_{i=1}^{Q} \sum_{j=1}^{s^{n}}\left(y_{i, j}-f_{i, j}\left(x_{i}, \mathrm{w}(k)\right)-J_{i, j} \Delta \mathrm{w}(k)\right)^{2}
$$

Taking the derivative with respect to $\triangle \mathrm{w}(\mathrm{k})$ and setting the result to zero gives

$$
\left(J(\mathrm{w}(k))^{T} J(\mathrm{w}(k))\right) \Delta \mathrm{w}(k)=J^{T}(\mathrm{w}(k))[y-f(\mathrm{w}(k))]
$$

Where $J(w(k))$ is the Jacobian matrix whose $i$,jth row equals $J_{i, j}(w(k)), f(w(k))$ and $y$ are vectors with $\mathrm{i}, \mathrm{jth}$ component $\mathrm{f}_{\mathrm{i}, \mathrm{j}}\left(\mathrm{x}_{\mathrm{i}}, \mathrm{w}(\mathrm{k})\right)$ and $\mathrm{y}_{\mathrm{i}, \mathrm{j}}$, respectively.

Thus, the increment vector of $\mathrm{w}(\mathrm{k})$ in each iteration step is

$$
\Delta \mathrm{w}(k)=\left(J^{T}(\mathrm{w}(k)) J(\mathrm{w}(k))\right)^{-1} J^{T}[y-f(\mathrm{w}(k))]
$$

Replacing $\mathrm{J}^{\mathrm{T}}(\mathrm{w}(\mathrm{k})) \mathrm{J}(\mathrm{w}(\mathrm{k}))$ with $\mathrm{J}^{\mathrm{T}}(\mathrm{w}(\mathrm{k})) \mathrm{J}(\mathrm{w}(\mathrm{k}))+\lambda \mathrm{I}$

$$
\Delta \mathrm{w}(k)=\left(J^{T}(\mathrm{w}(k)) J(\mathrm{w}(k))+\lambda I\right)^{-1} J^{T}(\mathrm{w}(k))[y-f(\mathrm{w}(k))]
$$

So update $\mathrm{w}(\mathrm{k})$

$$
\mathrm{w}(k+1)=\mathrm{w}(k)+\left(J^{T}(\mathrm{w}(k)) J(\mathrm{w}(k))+\lambda I\right)^{-1} J^{T}(\mathrm{w}(k))[y-f(\mathrm{w}(k))]
$$

Where $\mathrm{I}$ is the identity matrix and $\lambda$ is the damping factor. 


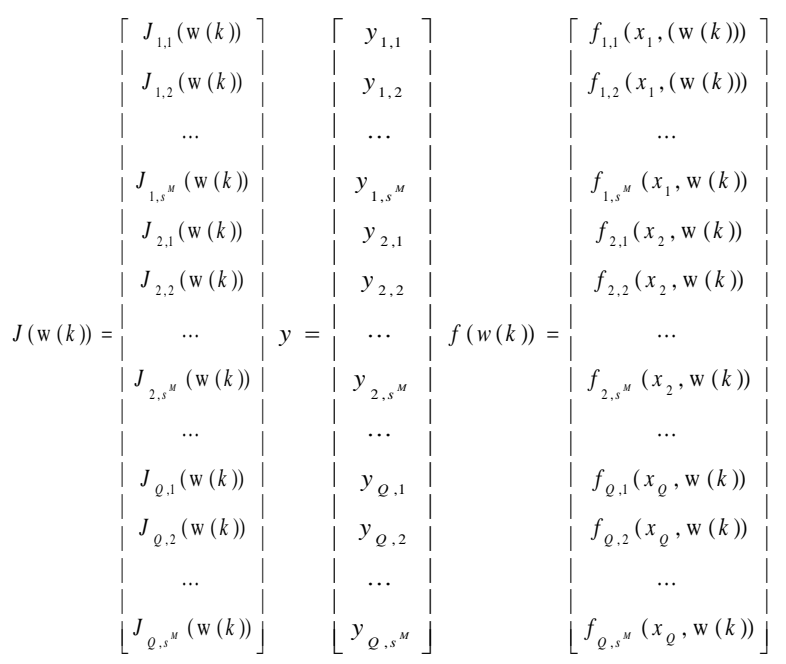

The algorithm can ensure fast training convergence by means of simplifying computation burden and meeting the requirement of all errors towards declination.

\section{ANN Design}

ANN has been widely used due to its highly nonlinear mapping nature, superior learning, generalization and recalling capability, and built-in fault tolerance. Fault types can be determined according to the relation matrix between feature inputs and fault outputs established by training the ANN. Here, a three-layer ANN with back propagation is designed to classify bearing conditions. The number of nodes in the input layer is varied from 3 to 5 , which represent the normalized features extracted from measured vibration signals. The number of neurons in the output layer is 4 corresponding to healthy, outer race defect, inner race defect, and ball defect which are represented by the output vector $\left[\begin{array}{llll}1 & 0 & 0 & 0\end{array}\right] \mathrm{T},\left[\begin{array}{llll}0 & 1 & 0 & 0\end{array}\right] \mathrm{T}$, $\left[\begin{array}{llll}0 & 0 & 1 & 0\end{array}\right] \mathrm{T}$, and $\left[\begin{array}{llll}0 & 0 & 0 & 1\end{array}\right] \mathrm{T}$, respectively. The ability of the ANN to adapt can be improved with increasing the number of neurons in the hidden layer. However, it leads to more calculation simultaneously. In order to attain appropriate response and reduce calculation, the number of neurons chosen for the hidden layer is 5. A sigmoid transfer function is selected for both the hidden layer and the output layer. The ANN is trained using the training algorithm described in section 3. Training stops and the latest vector $\mathrm{w}(\mathrm{k})$ is considered to be the optimum one if any of these conditions occurs: The maximum number of epochs is reached 5000. Performance has been minimized to the goal of 10-10. The gradient falls below the minimum value of 10-10. The efficiency of the ANN to identify defects is evaluated using test data.

\section{Results and Discussion}

As mentioned in section 2, 20 patterns were obtained from each channel for every bearing condition. Among these 20 patterns the first 12 patterns were used to train the ANN, the remaining 8 were used for test.

\subsection{Original Signal Analysis}

The ability of original signals to identify defects would be validated through the ANN. The input vector for the ANN was created as

$$
x=\left[\begin{array}{lllll}
R M S & \sigma^{2} & \gamma_{3} & \gamma_{4} & \gamma_{6}
\end{array}\right]^{T}
$$


The results in Table 1 show that the decision reached 100\% for training data and over $87 \%$ for test data using one channel signals. Hence, the selected channel positions were proper for bearing condition classification where the bearing defects had big influence on the vibration. For the group of channel DE and FE, the training ended because the minimum gradient rather than the performance goal was first met, which brought to $98.96 \%$ decision for training data and relatively low decision of $81.25 \%$ for test data. It was concluded that signals from one channel were superior to the group in diagnostic process.

\section{Table 1. Results of Original Signals in Bearing Condition Identification}

\begin{tabular}{clll}
\hline Channel & Training decision (\%) & Test decision (\%) & Epoch \\
\hline DE & 100 & 87.5 & 47 \\
FE & 100 & 93.75 & 27 \\
DE,FE & 98.96 & 81.25 & 54 \\
\hline
\end{tabular}

Table 2 demonstrates the influence of features on the identification of bearing condition. For all the cases, though the decision for training data remained beyond $90 \%$, the decision for test data kept declining with the reduction in number of features. When $\sigma 2$ was removed from input features extracted from channel FE, the test decision fell from over $90 \%$ to about $68 \%$ and the training completed till 84 iterations. For channel DE, the test decision indicated a steep descent by about $10 \%$ with the number of features decreasing, especially the lack of $\gamma 4$ or $\gamma 6$ made the drop even close to 20\%. The combination of FE and DE had similar effects. As a result, all five features should be considered to promote the diagnostic accuracy. Figure 3 shows the actual values of four outputs neurons for both training and test data from channel FE, respectively. Besides, convergence curves for this case are shown in Figure 4.

Table 2. Results of Features for Bearing Condition Identification

\begin{tabular}{|c|c|c|c|c|c|c|c|c|c|}
\hline \multirow[t]{2}{*}{ Features } & \multicolumn{3}{|c|}{ Training decision (\%) } & \multicolumn{3}{|c|}{ Test decision (\%) } & \multicolumn{3}{|c|}{ Epoch } \\
\hline & $\mathrm{DE}$ & $\mathrm{FE}$ & DE\&FE & $\mathrm{DE}$ & $\mathrm{FE}$ & DE\&FE & $\begin{array}{c}\text { D } \\
E\end{array}$ & $\mathrm{FE}$ & $\mathrm{DE} \& \mathrm{FE}$ \\
\hline$R M S \sigma^{2} \gamma_{3} \gamma_{4} \gamma_{6}$ & 100 & 100 & 98.96 & 87.5 & 93.75 & 81.25 & 47 & 27 & 54 \\
\hline$\sigma^{2} \gamma_{3} \gamma_{4} \gamma_{6}$ & 100 & 100 & 96.88 & 81.25 & 90.63 & 73.44 & 33 & 59 & 60 \\
\hline $\begin{array}{lll}\gamma_{3} & \gamma_{4} & \gamma_{6}\end{array}$ & 100 & $3^{95.8}$ & 90.63 & 71.88 & 68.75 & 75 & 70 & 84 & 99 \\
\hline$\sigma^{2} \gamma_{4} \gamma_{6}$ & 100 & 100 & 98.96 & 71.88 & 93.75 & 76.56 & 52 & 29 & 95 \\
\hline$\sigma^{2} \gamma_{3} \gamma_{6}$ & 100 & 100 & 91.67 & 62.5 & 90.63 & 56.25 & 85 & 20 & 55 \\
\hline$\sigma^{2} \gamma_{3} \gamma_{4}$ & 100 & 100 & 92.71 & 62.5 & 90.63 & 65.63 & 70 & 24 & 91 \\
\hline
\end{tabular}



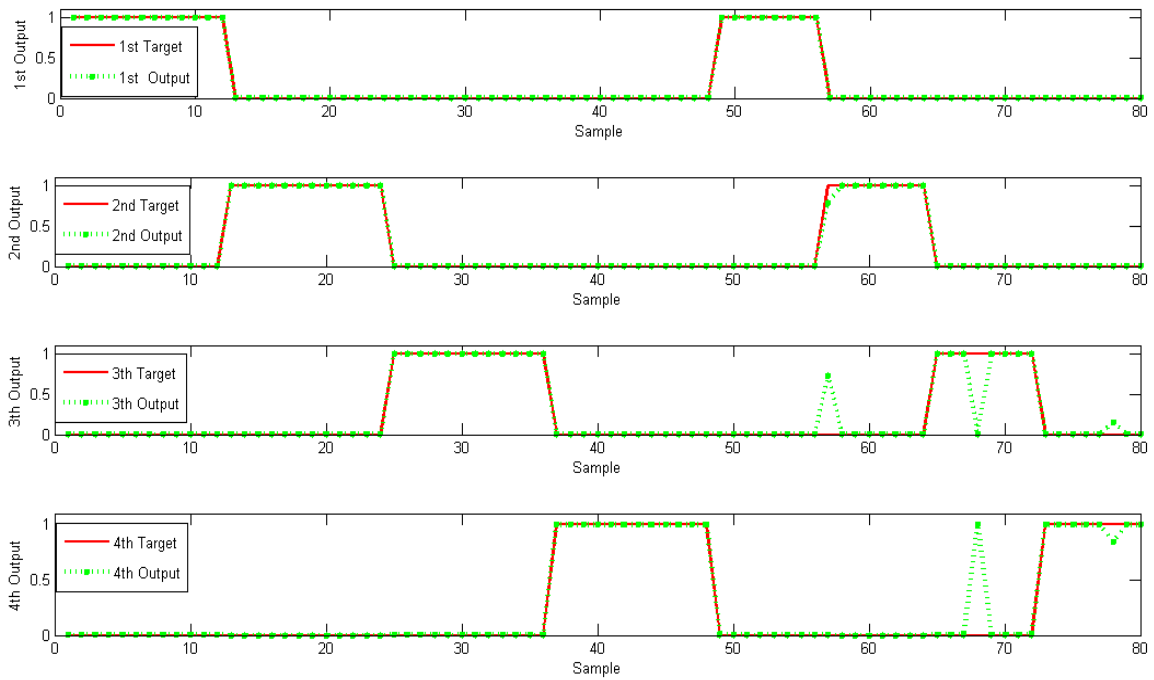

Figure 3. Targets and Actual Values of Four Output Nodes

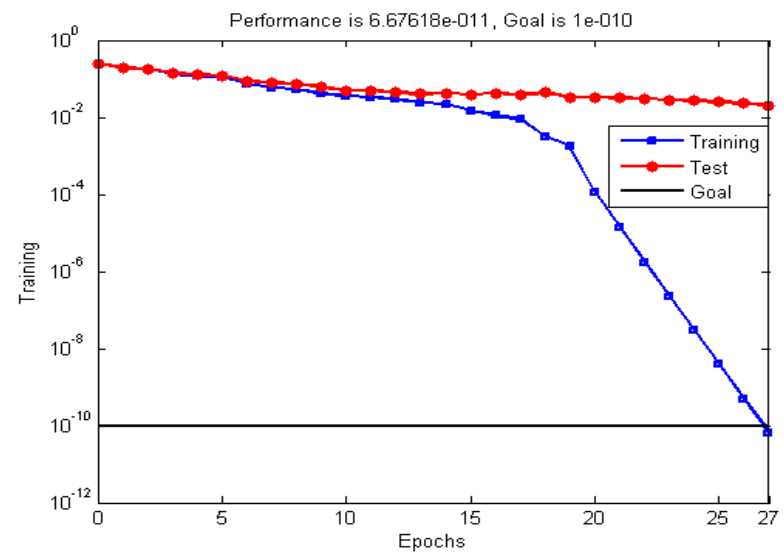

Figure 4. Convergence Learning Curve for Signal FE

\subsection{Filtered Signal Analysis}

The difficulty in detection of the defect lies in the fact that the signature of a defective bearing is spread across a wide frequency band excited by the impulses arising out of the lowfrequency bearing defects and hence can be easily masked by noise and low frequency interference generated by loads and environmental factors. For solving this problem, three band-pass (BP1-BP3) and one high-pass (HP) filters with finite-duration impulse response (FIR) were designed to suppress the low-frequency interference components. The band-pass frequencies (in $\mathrm{kHz}$ ) were chosen as: BP1 (3.0-9.0), BP2 (3.0-18), and BP3 (1.0-9.0). The cut-off frequency of the HP filter was chosen as $1.0 \mathrm{kHz}$. For channel FE, with healthy and defective bearings, before and after filtration using BP3, is shown in Figure 5.

Five time domain features were calculated from each of these filtered signals and used as inputs of the ANN. Table 3 shows the results for different channel signals and filters. The decision for training data was $100 \%$ in all cases. The decision for test data varied from $93.75 \%$ to $87.5 \%$. As a whole, the use of band-pass or high-pass filtration improved the 
decision by filtering the interfering signals outside the selected frequency scope, especially for channel DE and DE\&FE compared to that of original signals in Table 1. The effectiveness of the proposed features for the signal components in the selected band is verified.

\section{Table 3. Results of Filtered Signals for Bearing Condition Identification}

\begin{tabular}{cllll}
\hline Channel & Filter & Training decision (\%) & Test decision $(\%)$ & Epochs \\
\hline DE & BP1 & 100 & 87.50 & 31 \\
FE & BP1 & 100 & 87.50 & 31 \\
DE,FE & BP1 & 100 & 87.50 & 34 \\
FE & BP2 & 100 & 90.63 & 19 \\
DE & BP3 & 100 & 87.50 & 27 \\
\hline \multicolumn{7}{l}{ Frequencies (kHz): BP1 (3.0-9.0); BP2 (3.0-18); BP3 (1.0-9.0); HP (1.0) }
\end{tabular}
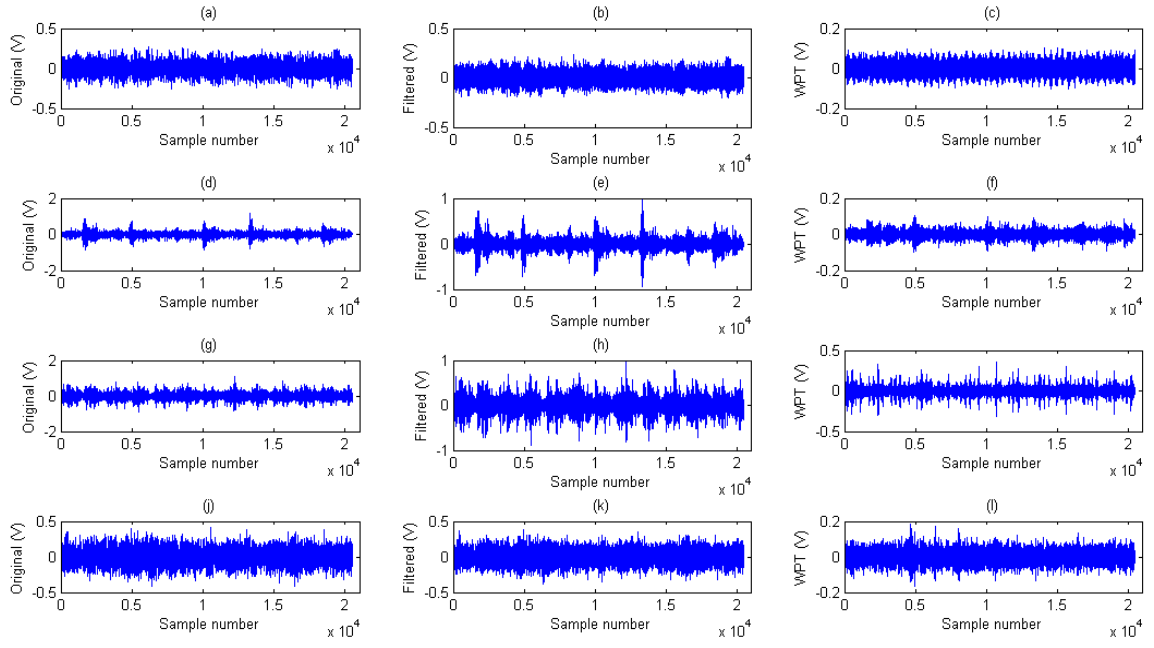

Figure 5. Time Domain Vibration Signal FE: First Row: Normal, Second Row: Outer Defect, Third Row: Inner Defect, Fourth Row: Ball Defect, First Column: Original, Second Column: band Pass Filtered (BP3), Third Row: Wavelet Transformed (6-9 kHz)

\subsection{Wavelet Packet Decomposition}

Wavelet packet (WPT) analysis, well suited for analyzing the non-stationary signals, is a generalization of wavelet decomposition offering a richer range of possibilities for signal analysis $[17,18]$. In this research, the acquired vibration signals were decomposed through WPT using Daubechies wavelet of order 8 (Db8) at level 3 to obtain approximation and detail coefficient vector, which again were reconstructed for features extraction. Figure 5 shows the reconstructed time domain signal in the range of $(6.0-9.0 \mathrm{kHz})$ for channel FE with healthy and defective bearings, respectively.

Table 4 shows the results for different channel signals decomposed by WPT. The features extracted from $(6.0-9.0 \mathrm{kHz})$ signals gave very good results $(96.88-100 \%)$ indicating the influence of the bearing defects on the components in the band $(6.0-9.0 \mathrm{kHz})$. The results show using WPT successfully isolates the components about the initial signal and the repetition period of the impact response from the rest of the overall vibration response of the 
bearing. Moreover, the application of WPT offered higher test decision than that of noprocessing, band-pass or high-pass filtration.

Table 4. Results of WPT Analysis for Bearing Condition Identification

\begin{tabular}{lllll}
\hline Channel & Details(kHz) & Training decision (\%) & Test decision (\%) & Epoch \\
\hline DE,FE & $3-6$ & 97.90 & 75 & 61 \\
DE & $6-9$ & 100 & 96.88 & 16 \\
FE & $6-9$ & 100 & 100 & 30 \\
DE,FE & $6-9$ & 100 & 96.88 & 36 \\
DE & $9-12$ & 100 & 84.38 & 28 \\
FE & $9-12$ & 100 & 100 & 17 \\
DE,FE & $9-12$ & 100 & 82.80 & 36 \\
DE,FE & $12-15$ & 100 & 87.50 & 28 \\
DE,FE & $15-18$ & 97.90 & 82.80 & 112 \\
DE,FE & $18-21$ & 96.88 & 40.63 & 81 \\
\hline
\end{tabular}

\subsection{Envelope Analysis}

The spectrum of a defective bearing consists of harmonics of the CDF with the highest amplitude around the mechanical system resonance frequency. However, the amplitude at the $\mathrm{CDF}$ is small and not easily observed. Envelope analysis can be performed by digitizing the signal and band pass filtering it in a region where there is a high signal-to-noise ratio, typically around a structural resonance [19]. The filtered signal is then demodulated to eliminate the carrier frequencies. Both signal components in the range of $(6.0-9.0 \mathrm{kHz})$ and (9.0-12 kHz) obtained in subsection 5.3 were used for carrying out the envelope analysis. The instantaneous amplitudes of each signal components were calculated using Hilbert transform (HT), and these instantaneous amplitudes of time series made up of the envelopes [20, 21]. The features extracted from the envelopes were used to train and test the ANN. The results are shown in Table 5. The results are nearly similar to that of the signals in Table 4. The major improvement in channel DE and DE\&FE compared to that of original and other processed signals may be attributed to the better discrimination of the envelope features with different conditions in the bearings. The results also show the effects of interfering signals within the selected frequency band can be minimized using envelope analysis.

Table 5. Results of Envelope Analysis for Bearing Condition Identification

\begin{tabular}{cllll}
\hline Channel & Details(kHz) & Training decision (\%) & Test decision (\%) & Epoch \\
\hline DE & $6-9$ & 100 & 96.88 & 23 \\
FE & $6-9$ & 100 & 96.88 & 50 \\
DE,FE & $6-9$ & 100 & 90.63 & 40 \\
DE & $9-12$ & 100 & 96.88 & 33 \\
FE & $9-12$ & 100 & 100 & 50 \\
DE,FE & $9-12$ & 100 & 95.30 & 45 \\
DE,FE & $12-15$ & 100 & 89.06 & 52 \\
\hline
\end{tabular}

Table 6 shows a comparison between the present work and four published works using different features generated from the vibration signal of rotating machinery and ANN for classification. All eight methods attain a high classification rate, but efficiency of these methods is different from each other. In [15], statistical parameters were used to identify only between healthy and faulty bearings. In $[8,22]$, the authors used spectral features which needs rapid processing, high accuracy, and a large quantity of complicated calculations compared to 
the features obtained using zero-crossing [23] and the presented approaches. Besides the common simplicity for feature extraction directly from time domain signals without complex analysis both the presented approaches and zero-crossing, the proposed features related to WPT and envelope are slightly better than the features obtained using zero-crossing for classification.

Table 6. Comparison between Eight Methods for Identifying Bearing Faults

\begin{tabular}{llll}
\hline Reference & Feature & condition & decision \\
\hline $\begin{array}{l}\text { Malhhi and Gao [20] } \\
\text { Samanta and AI-Balushi }\end{array}$ & Wavelet + PCA & Outer, inner defect & $82.8-96.3$ \\
$\begin{array}{l}\text { [15] } \\
\text { Li et al.[8] }\end{array}$ & Normal and defective & $98-100$ \\
William and Hoffman [21] & Zero-crossing & Normal, outer, inner, ball defect & 86.8-96.2 \\
Present work & Original+ statistical & Normal, outer, inner, ball defect & $91.5-97.1$ \\
& FIR+ statistical & Normal, outer, inner, ball defect & $87.5-93.8$ \\
& WPT+ statistical & Normal, outer, inner, ball defect & $90.6-93.8$ \\
& Envelope+ statistical & Normal, outer, inner, ball defect & $96.9-100$ \\
\hline
\end{tabular}

\section{Conclusions}

A model for detection and identification of bearing faults using time domain features is explored. The results show all four different methods achieve a high classification rate. Using these time domain features not only leads to a high rate of almost $100 \%$ success for training data and over $90 \%$ success for test data, but also results in a low computational burden with less than 50 training epochs. Low training epochs should attribute to small number of features, fast training algorithm, and processed vibration signals. Signals from channel FE and all five features selected as inputs of ANN are better than others for classification. Features acquired from WPT and envelope analysis are also slightly better than other features in identification of bearing faults. In consequence, using time domain features combined with ANN is efficient for identification of bearing faults.

\section{Acknowledgments}

The work was supported by National High Technology Research and Development Program of China (2009AA11Z217).

\section{References}

[1] Benbouzie and M.E.H, A review of induction motors signature analysis as a medium for faults detection, IEEE Transactions on Industrial Electronics, vol.47, (2000), pp.984-993.

[2] G.-m. Xian, Mechanical failure classification for spherical roller bearing of hydraulic injection molding machine using DWT-SVM, Expert Systems with Applications, vol.37, (2010), pp.6742-6747.

[3] S. Nandi, H. A. Toliyat and L. Xiaodong, "Condition monitoring and fault diagnosis of electrical motors-a review”, IEEE Transactions on Energy Conversion, vol.20, (2005), pp.719-729.

[4] N. Tando and A. Choudhury, "A review of vibration and acoustic measurement methods for the detection of defects in rolling element bearings", Tribology International, vol.32, (1999), pp. 469-480.

[5] J. Zarei and J. Poshtan, "Bearing fault detection using wavelet packet transform of induction motor stator current", Tribology International,vol. 42, (2007), pp.213-219.

[6] S. Nandi, H.A. Toliyat and X. Li, Condition monitoring and fault diagnosis of electrical motors-a review, IEEE Trans. Energy Convers, vol.20, (2005), pp.719-729.

[7] W. R. Finley, M. M. Hodowanec and W. G. Holter, "An analytical approach to solving motor vibration problems”, IEEE Transactions on Industry Applications, vol.36, no. 5, (2000), pp.1467-1480. 
[8] B. Li, M. Y. Chow, Y. Tipsuwan and J. C. Hung, "Neural-network-based motor rolling bearing fault diagnosis", IEEE Transactions on Industrial Electronics, vol.47, no.5, (2000), pp.1060-1069.

[9] V. K. Rai and A. R. Mohanty, "Bearing fault diagnosis using FFT of intrinsic mode functions in HilbertHuang transform", Mechanical Systems and Signal Processing, vol.21, (2007), pp.2607-2615.

[10] J. Zarei and J. Poshtan, "An advanced Park's vectors approach for bearing fault detection”, Tribology International, vol.42, (2009), pp.213-219.

[11] C. T. Yiakopoulos, K. C. Gryllias and I. A. Antoniadis, "Rolling element bearing fault detection in industrial environments based on a K-means clustering approach", Expert Systems with Applications, vol.38, (2011), pp.2888-2911.

[12] R. Yan, R.X. Gao and C. Wang, Experimental evaluation of a unified time-scale-frequency technique for bearing defect feature extraction", ASME J. Vib.Acoust, vol.131, no.4, (2009).

[13] C.-C. Wanga, Y. Kang, P.-C. Shen, Y.-P. Chang and Y.-L. Chung, "Applications of fault diagnosis in rotating machinery by using time series analysis with neural network", Expert Systems with Applications, vol.37, (2010), pp.1696-1702.

[14] Raflee, P. W. Tse, A. Harifi and M. H. Sadeghi, "A novel technique for selecting mother wavelet function using an intelligent fault diagnosis system", Expert Systems with Applications, vol.36, (2009), pp.48624875 .

[15] B. Samanta and K. R. Al-Balushi, "Artificial neural network based fault diagnostics of rolling element bearings using time-domain features", Mechanical Systems and Signal Processing, vol.17, no.2, (2003), pp.317-328.

[16] J. Zarei, "Induction motor bearing fault detection using pattern recognition techniques", Expert Systems with Applications, vol.39, (2012), pp.68-73.

[17] N. G. Nikolaou and I. A. Antoniadis, "Rolling element bearing fault diagnosis using wavelet packets", NDT\&E International, vol.35, (2002), pp.197-205.

[18] S. Abbasion, A. Rafsangani, A. Farshidianfar and N. Irani, "Rolling element bearings multi-fault classification based on the wavelet denoising and support vector machine", Mechanical Systems and Signal Processing, vol.21, (2007), pp.2933-2945.

[19] P. W. Tse, Y. H. Peng and R. Yam, "Wavelet analysis and envelope detection for rolling element bearing fault diagnosis - Their effectiveness and flexibilities", Journal of Vibration and Acoustics, vol.123, (2001), pp.303-310.

[20] W. He, Z. Jiang and K. Feng, "Bearing fault detection based on optimal wavelet filter and sparse code shrinkage", Measurement, vol.42, (2009), pp.1092-1102.

[21] V.N. Patel, N. Tandon and R.K. Pandey, "Defect detection in deep groove ball bearing in presence of external vibration using envelope analysis and Duffing oscillator", Measurement, vol.45, no.5, (2012), pp.960-970.

[22] A. Malhi and R.X. Gao, "PCA-based feature selection scheme for machine defect classification", IEEE Trans. Instrum. Meas, vol.53, (2004), pp.1517-1525.

[23] P. E. William and M. W. Hoffman, "Identification of bearing faults using time domain zero-crossings", Mechanical Systems and Signal Processing, vol.25, (2011), pp.3078-3088. 